\title{
Diplomacia Pública Digital: el contexto iberoamericano
}

\author{
Daniel Aguirre, Matthias Erlandsen, Miguel Ángel López, EdS. \\ Escuela de Relaciones Internacionales de la Universidad Nacional de \\ Costa Rica, 2018 \\ 310 páginas
}

omo quizás una afortunada coincidencia, mientras se redachan anunciado en Twitter que han alcanzado un entendimiento sobre los principales puntos de las negociaciones comerciales entre ambos países ${ }^{1}$. Exactamente un año antes de esto, la viabilidad de cualquier acuerdo se había puesto en tela de juicio, también en dicha ocasión, debido a declaraciones en Twitter ${ }^{2}$. Este ejemplo ilustra tan solo uno de tantos casos donde mandatarios, cancilleres y otros actores preponderantes de la diplomacia, han utilizado las plataformas digitales en un mundo cada vez más interconectado y donde la información fluye a velocidades nunca antes vistas. Y, no obstante, pese a esta realidad, el uso de plataformas digitales por estos actores no se ha estudiado de forma sistemática, como bien señalan Aguirre, Erlandsen y López. Es debido a este vacío de análisis, que los editores buscan no tanto generar un consenso sobre conceptos, principios o parámetros para evaluar la denominada "diplomacia pública digital", sino el iniciar un diálogo que permita entender la evolución y estado actual de esta práctica en América Latina y España. A través de una compilación de textos que discu-

1 Peña Nieto, Enrique (@EPN) “He hablado con el Presidente @realDonaldTrump. México y Estados Unidos han alcanzado un entendimiento comercial. Deseamos la reincorporación de Canadá a las pláticas para lograr una exitosa negociación trilateral del TLCAN esta misma semana." 27/08/20I8, I0;44 AM. Tweet. Trump, Donald J (@realDonaldTrump) ”A big deal looking good with Mexico!" 27/08/20I8, 08:39 AM.Tweet.

Trump, Donald J (@realDonaldTrump) ”United States-Mexico Trade Agreement:!" 27/08/20I8, OI:II PM.Tweet

2 Trump, Donald J (@realDonaldTrump) "We are in the NAFTA (worst trade deal ever made) renegotiation process with Mexico \& Canada. Both being very difficult, may have to terminate?" 27/08/2017, 08:5r AM.Tweet 
ten tanto el uso institucional como también el personal de Estados y mandatarios, de las herramientas digitales, y en particular de Twitter, los autores han elaborado una obra que ofrece un compendio ideal para quienes se acercan, por primera vez, al estudio de esta transformación en la esfera diplomática iberoamericana.

Como ejemplo de este esfuerzo, el texto de Ramos y Espinoza es no solo relevante para el conjunto de la obra, sino quizá una buena introducción al mismo. Al analizar el uso que hacen representaciones y Embajadores mexicanos selectos del uso de Twitter para posicionar una agenda, expandir la presencia de dichos actores e insertarse eficientemente en un verdadero diálogo digital, los autores proveen al lector un marco conceptual para entender la diplomacia pública y proponen, además, algunos criterios para evaluar el estado de la misma en la Cancillería mexicana. Concluyen, muy acertadamente, que el potencial de estas herramientas y en particular de Twitter, está en "unir a los usuarios a la conversación” y, por ende, hacen un llamado a que el uso de estas herramientas por parte de la diplomacia mexicana pase de un fin primordialmente informativo, a buscar insertarse y generar una verdadera conversación global.

Usando una metodología diferente y centrando su análisis en un único actor, el texto de Elórtegui y Miles continúa el análisis institucional de la diplomacia pública, al demostrar el uso claramente intencional que hizo de su cuenta de Twitter el ex Ministro de Relaciones Exteriores de Ecuador, Ricardo Patiño. De manera metódica, los autores analizan el desempeño de la cuenta de Twitter del ex Ministro para arribar a la conclusión de que Ecuador, reconociendo el potencial de plataformas como Twitter, usa la voz digital de estos actores como una herramienta que abone a sus objetivos de política exterior. A diferencia del análisis de México previamente referido, los autores no buscan necesariamente evaluar el desempeño en sí de las herramientas, sino evidenciar el hecho de que, para el actor en cuestión, el uso de Twitter formaba parte de su diseño estratégico de comunicación gubernamental.

Concluyendo el primer segmento, y en contraste con el aná- 
lisis mayoritariamente cuantitativo de sus antecesores, Juan Luis Manfredi y Rafael Rubio proveen un resumen detallado del trabajo realizado en España en torno al entendimiento, evolución y estado actual de la diplomacia pública, incluido el arribo de la diplomacia digital en dicho país. Al ilustrar los objetivos que ha perseguido la diplomacia española con las herramientas disponibles en cada etapa, y cómo en dicho andamiaje se ha tratado de hacer que las nuevas tecnologías sirvan a los objetivos de política exterior, los autores permiten al lector comprender que las herramientas, por más novedosas que sean, deben tener un fin claro y acorde a las necesidades de sus operadores. En esta lógica, prevén que el futuro de esta diplomacia deberá ayudar a una verdadera transformación del quehacer diplomático y buscar incrementar la cercanía, la confianza y la transparencia del servicio público que prestan. Objetivos que, sin duda, como hemos visto desde Twitter, se pueden lograr con estrategias de uso óptimo de la plataforma que abonan a la salud de la conversación pública.

Superado el análisis institucional, la obra incorpora dos textos mediante los cuales los autores buscan evidenciar prácticas específicas y hacer conclusiones sobre el impacto que tuvieron las personalidades - no tanto las instituciones - en el uso de Twitter como herramienta de diplomacia pública. En concreto, la utilización que dieron a la red los ex Mandatarios de Venezuela y Argentina. Sobre estos textos, corresponde a cada lector extrapolar las mejores y peores prácticas en cada caso, pero el común denominador de ambas es el hecho de que dichos Mandatarios imprimieron su particular punto de vista en el manejo público de su voz en Twitter. En este tenor, los ejemplos ilustrados son relevantes y los autores hacen un excelente trabajo al indicar cómo dichas interacciones abonaron o no a una agenda estratégica.

Hilando una discusión lógica, los editores culminan la obra con la selección de textos que analizan contextos y casos de estudio específicos, donde el uso de Twitter coadyuvó a la implementación de estrategias de diplomacia digital. El lector encontrará en estos textos que analizan casos específicos en Brasil, Bolivia, Costa Rica y Chile, análisis detallados de las estructuras existentes que definieron la utilización de 
la diplomacia digital, pero quizás más importante aún de los contextos específicos que detonaron su utilización. Esto es, sin duda, muy relevante, ya que Twitter "es lo que está pasando" y, por ende, quienes desean implementar estrategias de comunicación en dicho espacio, requieren primero tener claridad de qué está sucediendo, para entonces poder conectar con los intereses de los twiteros. Resalta también el reconocimiento específico que se hace en varias partes a la plusvalía que traen herramientas como Twitter, sin importar el tamaño y recursos del actor, como señalan Méndez-Coto y Hernández “...los medios digitales y las redes sociales consisten en una herramienta que ha democratizado la comunicación y permitirá a estos países competir en la arena de la información" (pp 307).

A la luz de los diversos textos incluidos y las aportaciones de cada caso, el lector no podrá más que arribar a la conclusión deseada por los actores: la diplomacia pública digital es ya una realidad en América Latina. No obstante, hace falta mucho más trabajo para sistematizarla, ampliarla y llevarla a los alcances que permite hoy un mundo interconectado por la tecnología. Invito a quienes emprendan este texto buscando emular mejores prácticas, que harán bien en entender que Twitter es utilizado de forma más óptima por quienes entienden que la plataforma es un puente entre ciudadanos (@) y sus intereses (\#), no un megáfono. En consecuencia, quienes lo utilizan como este último, cumplen un mero fin informativo y pierden de vista la posibilidad de una estrategia holística de comunicación.

\section{POR MPA Hugo Rodríguez Nicolat}

Director de Política Pública de Twitter para

América Latina de Habla Hispana 